\title{
$N$-methyl- $N$-nitrosourea-induced changes in epithelial rests of Malassez and the development of odontomas in rats
}

\author{
AYAKO KIMURA $^{1 *}$, KATSUHIKO YOSHIZAWA ${ }^{1 *}$, TOMO SASAKI $^{1,2}$, NORIHISA UEHARA ${ }^{1}$, \\ YUICHI KINOSHITA ${ }^{1,3}$, HISANORI MIKI ${ }^{1}$, TAKASHI YURI ${ }^{1}$, TAKASHI UCHIDA ${ }^{4}$ and AIRO TSUBURA ${ }^{1}$ \\ ${ }^{1}$ Department of Pathology II, Kansai Medical University, Morguchi, Osaka 570-8506; \\ ${ }^{2}$ Kyoto R \& D Center, Maruho Co., Ltd., Kyoto, Kyoto 600-8815; ${ }^{3}$ Division of Pathology, \\ Kansai Medical University Takii Hospital, Morguchi, Osaka 570-8505; ${ }^{4}$ Department of Oral Biology, \\ Hiroshima University Graduate School of Biomedical Science, Hiroshima 734-8553, Japan
}

Received February 9, 2012; Accepted April 4, 2012

DOI: $10.3892 /$ etm.2012.559

\begin{abstract}
Morphological changes in the epithelial rests of Malassez (ERM) and the development of odontogenic tumors in the molars of female Lewis rats treated at 4 weeks of age with a single intraperitoneal injection of $50 \mathrm{mg} / \mathrm{kg}$ of $N$-methyl$N$-nitrosourea (MNU) were examined at 12, 18 and 30 weeks of age. Following MNU exposure, the total number and average area of ERM in the cervical and furcational regions of the first, second and third molars of the mandible and maxilla were compared with age-matched control animals. The number of ERM at each time point was significantly greater in the MNU-treated group compared to the control group, but there was no time-dependent increase in the number of ERM in either group. The area of ERM was significantly larger in the MNU-treated group compared to the control group at each time point, and it increased in a time-dependent manner in the MNU-treated group. No increases in the number or area of ERM were observed in the control group. At 30 weeks of age, $23 \%$ of the MNU-treated rats had developed odontomas (complex type) in the molar region as well as in the incisor region. Immunohistochemically, the expression of tyrosine receptor kinase A (TrkA) and cytokeratin 14 (CK14) decreased, whereas p63 expression remained high during ERM enlargement. In tumors, ameloblast-like cells were positive for amelogenin, TrkA and CK14 but negative for p63, whereas odontoblast-like cells were negative for all antigens examined. In conclusion, a single intraperitoneal injection of MNU
\end{abstract}

Correspondence to: Dr Katsuhiko Yoshizawa, Department of Pathology II, Kansai Medical University, Morguchi, Osaka 570-8506, Japan

E-mail: yoshizak@takii.kmu.ac.jp

${ }^{*}$ Contributed equally

Key words: amelogenin, cytokeratin 14, epithelial rests of Malassez, $N$-methyl- $N$-nitrosourea, odontogenic tumor, p63, tyrosine receptor kinase A caused the development of odontomas in the molar region; these tumors were possibly derived from ERM.

\section{Introduction}

Rodent teeth are sensitive to chemicals and are good models for the evaluation of chemically induced effects in humans. However, their biological features, particularly those of the incisors, are quite different from those of human teeth $(1,2)$. Rats have 16 teeth, comprising an incisor and three molars in each quadrant. Rodent incisors grow, calcify and erupt continuously throughout life. A longitudinal section shows the complete life cycle of tooth development from inception to maturity $(3,4)$. The frequency of odontogenic tumors is low in rodents (5) and humans. The odontogenic epithelium is responsible for tooth development under physiological conditions and can give rise to tumors and cysts in the jaw $(6,7)$. Therefore, tumors derived from the perpetually erupting ameloblasts of the rodent incisor may differ from those in human adults. The chemical compounds that cause morphological changes in rodent teeth include various antitumor or DNA-alkylating agents, such as 5-fluorouracil, doxorubicin, mitomycin $\mathrm{C}$, vinblastine sulphate, docetaxel, irinotecan hydrochloride, cisplatin and nitrosoureas. These compounds cause a variety of dental lesions in the incisors and/or molars (5,8-10). Among these chemicals, $N$-ethyl- $N$-nitrosourea (ENU) and $N$-methyl$N$-nitrosourea (MNU) have deleterious effects on odontogenic tissues, resulting in tooth deformation and malocclusion and eventually odontogenic tumors in rats $(9,11-17)$ and hamsters $(8,15,18-20)$.

Epithelial rests of Malassez (ERM) were first described by Malassez in 1884. In studies of human teeth, Malassez noted that ERM form a network around the tooth root and that the number of ERM decreases with age (21). ERM are quiescent epithelial remnants of Hertwig's epithelial root sheath that remain in the periodontal ligament throughout life (22-24); the exact function of these structures has not been clarified. ERM subcultured with primary dental pulp cells can differentiate into ameloblast-like cells and generate enamel-like and/or dentin-like tissues (25). ERM have the potential to proliferate 
in response to appropriate extracellular signals such as mitogens, and the proliferation of ERM may lead to the formation of odontogenic tumors or cysts $(26,27)$. However, the changes that occur in ERM during the process of odontogenic tumor development have not been closely examined. The present study focuses on the changes in ERM during the development of odontomas in the molar region and the morphological and immunohistochemical characterization of these lesions induced by a single intraperitoneal injection of MNU into female Lewis rats.

\section{Materials and methods}

Animals. A total of 43 3-week-old female Lewis rats [LEW/ CrlCrlj] were purchased from Charles River Japan (Atsugi, Japan). All rats were housed 4-5 in a plastic cage with paper bedding (Paper Clean, SLC, Hamamatsu, Japan) in a temperature- $\left(22 \pm 2^{\circ} \mathrm{C}\right)$ and humidity- $(60 \pm 10 \%)$ controlled animal room under a 12-h light/dark cycle.

Experimental procedures. After an acclimatization period of 1 week, rats were divided into two groups. A total of 25 rats received an intraperitoneal injection of $50 \mathrm{mg} / \mathrm{kg} \mathrm{MNU}$ (Sigma, St. Louis, MO, USA). MNU was dissolved in physiologic saline containing $0.05 \%$ acetic acid immediately prior to injection. The control group consisted of 18 rats that were injected with vehicle only (physiologic saline containing $0.05 \%$ acetic acid). All rats were fed a commercial pellet diet (CMF $30 \mathrm{kGy}$, Oriental Yeast, Chiba, Japan) and had ad libitum access to water throughout the experiment. During the experiments, clinical signs were observed once a day, and body weight was measured once a week. At 12 and 18 weeks of age, 5 randomly selected rats in the control group and 6 in the MNU-treated group were sacrificed. The remaining rats in the control group $(n=8)$ and the MNU-treated group $(n=13)$ were sacrificed at 30 weeks of age. Complete necropsies were conducted on all animals. All procedures involving animals were approved by the Animal Experimentation Committee of Kansai Medical University.

Tissue sampling. The rats were anesthetized with isoflurane (Forane ${ }^{\circledR}$; Abbot Japan, Tokyo, Japan), and the left and right sides of the mandible and maxilla were separately dissected from the surrounding tissues. The collected tissue was immersed in $10 \%$ neutral buffered formalin for one week and demineralized in a 10\% EDTA solution ( $\mathrm{pH}$ 7.0-7.3; Osteosoft ${ }^{\circledR}$, Merk KGaA, Darmstadt, Germany) at room temperature for 4-6 weeks. The samples were then dehydrated with graded ethanol and embedded in paraffin. Sagittal sections were cut in the mesiodistal direction and included the first, second and third molars and the incisors, as previously described (28). Sections were stained with hematoxylin and eosin (H\&E) or used for immunohistochemistry. ERM of the first, second, and third molars and the induced tumors were histopathologically evaluated. Histopathological and immunohistochemical evaluations were reviewed by a toxicologic pathologist certified by the Japanese Society of Toxicologic Pathology and/or by the International Academy of Toxicologic Pathology (K.Y. and A.T.), according to the previously defined histopathological terminology and diagnostic criteria $(2,29,30)$.
Immunohistochemistry. The labeled streptavidin biotin (LSAB) technique was performed with an LSAB staining kit (Dako, Carpinteria, CA, USA). The following antibodies were used: rabbit anti-tyrosine receptor kinase A (TrkA) polyclonal antibody (sc-118, 1:50 dilution; Santa Cruz Biotechnology, Santa Cruz, CA, USA) as a nerve growth factor receptor in periodontal ligament epithelium $(31,32)$; mouse anti-human CK14 monoclonal antibody (clone LL002, 1:20 dilution; Leica, Microsystems, Wetzlar, Germany) as a basal cell keratin marker (25); mouse anti-human p63 monoclonal antibody (clone 4A4, 1:50 dilution; Dako, Glostrup, Denmark) as an epithelial stem cell marker in oral tissue (33-38); and rabbit anti-porcine amelogenin polyclonal antibody (raised by T.U., 1:5000 dilution) $(39,40)$ as an ameloblast marker $(41,42)$. Each primary antiserum or antibody was incubated overnight at $4{ }^{\circ} \mathrm{C}$ without antigen retrieval. The reaction products were visualized using 3-3'-diaminobenzidine tetrahydrochloride.

Morphometric analysis. H\&E-stained sections of the jaw were scanned with a high-resolution digital slide scanner (NanoZoomer 2.2 Digital Pathology, Hamamatsu Photonics, Hamamatsu, Japan) to prepare digital images. The ndpi image files were opened in color mode with NDP.view software (Hamamatsu Photonics), and the area of ERM was measured in both sides of the maxillary and mandibular jaws; the number of ERM was counted in the cervical and furcation regions of the first, second, and third molars of the mandible and maxilla. An experimental dentist (A.K.) performed morphometric analysis using Image J Windows software (National Institutes of Health, Bethesda, MD, USA).

Statistical analysis. All discrete values are expressed as mean \pm standard error (SE) and were analyzed with the two-tailed independent Student's t-test for unpaired samples after confirming the homogeneity of variance. The statistical analysis was used to examine the significance of differences in the number and the area of ERM between MNU-treated rats and control rats at each time point and between different time points. P-values $<0.05$ were considered to indicate statistical significance.

\section{Results}

General remarks. None of the animals died during the study period. Body weight gain was lower in the MNU-treated rats than in the control rats (data not shown). By the age of 30 weeks, the MNU-treated rats developed mammary tumors (data not shown).

Morphological analysis of proliferative changes in molars. In the MNU-untreated control rats, ERM were observed in the cervical and furcation regions of the first, second, and third mandibular and maxillary molars and were characterized by a high nuclear/cytoplasmic ratio and condensed nuclei (Fig. 1a). Moreover, the size of the ERM remained small, and there were no neoplastic changes in the periodontal ligament at any time point. The ERM in MNU-treated animals at 12, 18 , and 30 weeks of age had similar morphology to the ERM in control rats, but the size of the ERM gradually increased after MNU treatment (Fig. 1b). No odontogenic tumors were 


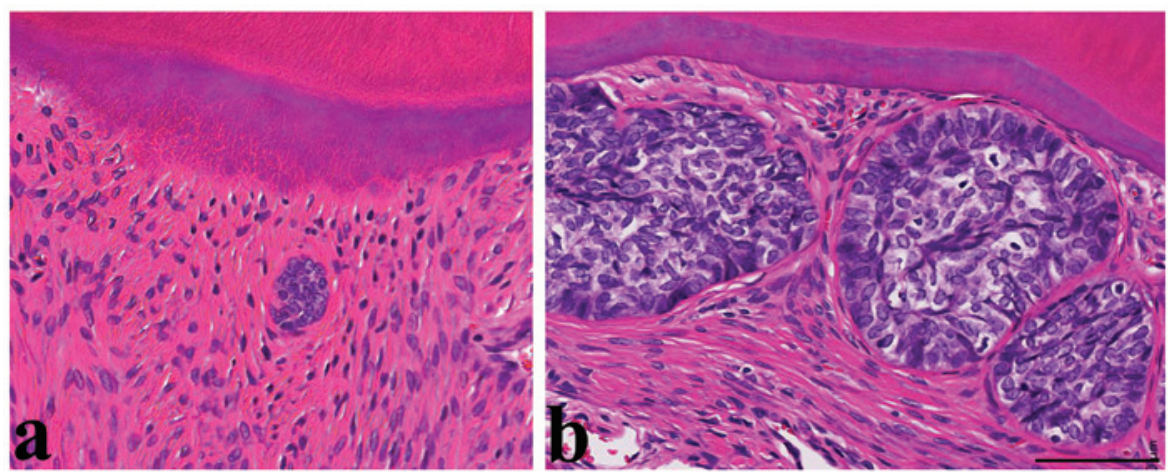

Figure 1. Epithelial rests of Malassez (ERM). (a) Small ERM composed of cells with high nuclear-cytoplasmic ratios are found in a 30-week-old control rat. (b) ERM in a 30-week-old MNU-treated rat are large, however, the morphology is similar to that of ERM in the control rat. H\&E staining; bar, $100 \mu \mathrm{m}$.
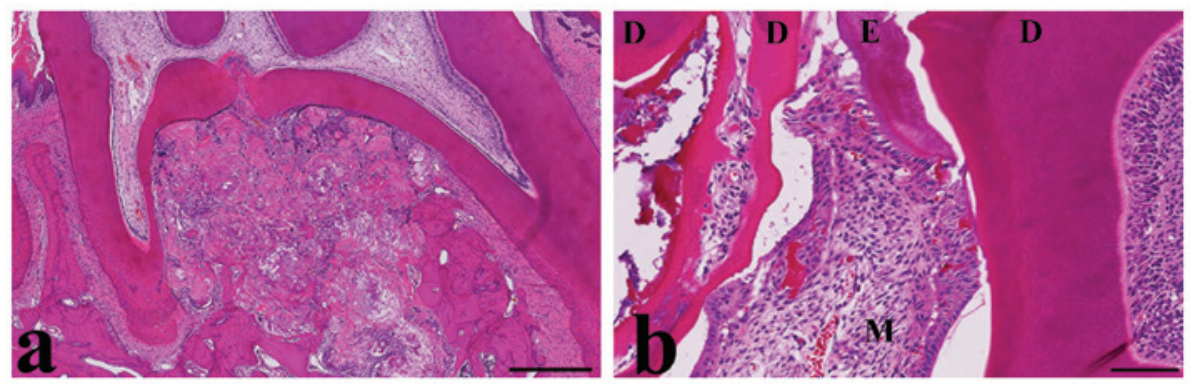

Figure 2. An $N$-methyl- $N$-nitrosourea (MNU)-induced odontogenic tumor. (a) The tumor is located in the furcational region of the second right maxillary molar. H\&E staining, bar, $500 \mu \mathrm{m}$. (b) The tumor is composed of (D) dentin-like and (E) enamel-like tissues together with (M) dental pulp-like mesenchymal cells, suggestive of a complex odontoma. H\&E staining; bar, $100 \mu \mathrm{m}$.

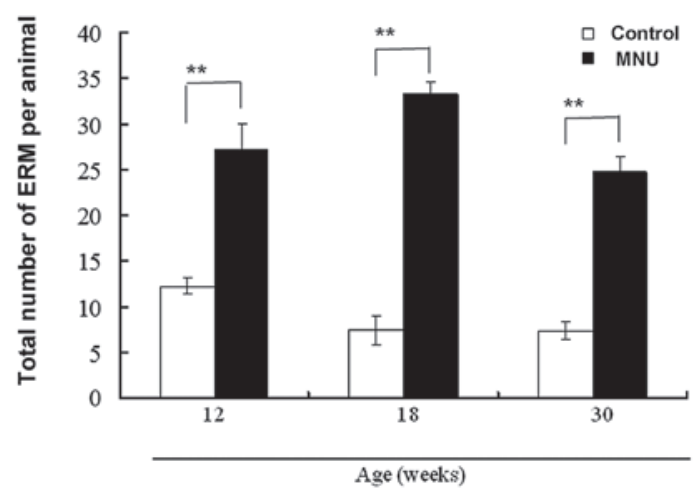

b

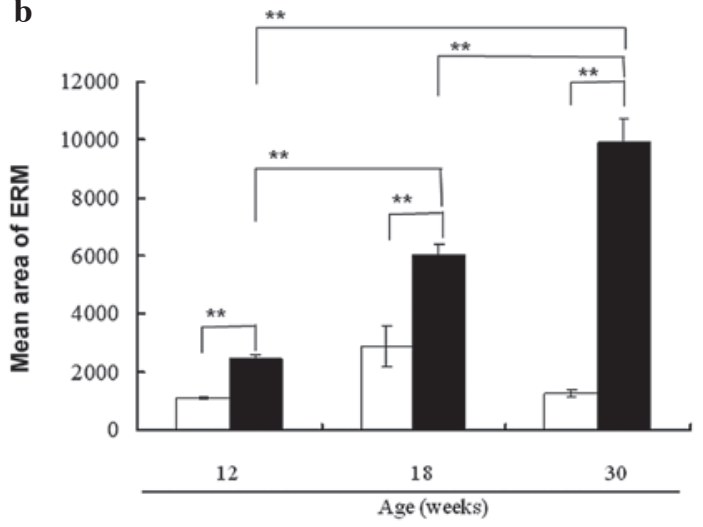

Figure 3. Time-course progression of the number and area of epithelial rests of Malassez (ERM) in the mandibular jaws of control and $N$-methyl- $N$-nitrosourea (MNU)-treated rats. (a) Total number of ERM per rat was significantly higher in the MNU-treated animals than in the control animals at each time point, but the number did not increase in a time-dependent manner. (b) Mean area $\left(\mu \mathrm{m}^{2}\right)$ of ERM was significantly higher in the MNU-treated animals at each time point, and the area increased in a time-dependent manner. Means \pm SE are shown. ${ }^{* *} \mathrm{P}<0.01$.

detected at 12 or 18 weeks of age. At 30 weeks of age, odontogenic tumors were found in three of 13 MNU-treated rats (23\% incidence). These tumors were located near the third mandibular molars. Two odontogenic tumors appeared in the cervical and furcation regions, indicating molar origin (Fig. 2a). These tumors contained a mixture of ameloblast-like cells with enamel-like tissue, odontoblast-like cells with multinucleated giant cells and dentin-like tissue, and dental pulp-like mesenchymal cells (Fig. 2b). The third tumor had similar morphology. It was difficult to determine the origin of this tumor, but it was located at the base of the incisor, indicative of incisor origin. The morphology of the three MNU-induced tumors was indicative of odontoma (complex type).

Morphometrical analysis of epithelial rests of Malassez (ERM). The number of ERM per rat remained low in control rats (12.2, 7.4, and 7.3 at 12,18 , and 30 weeks, respectively), but 

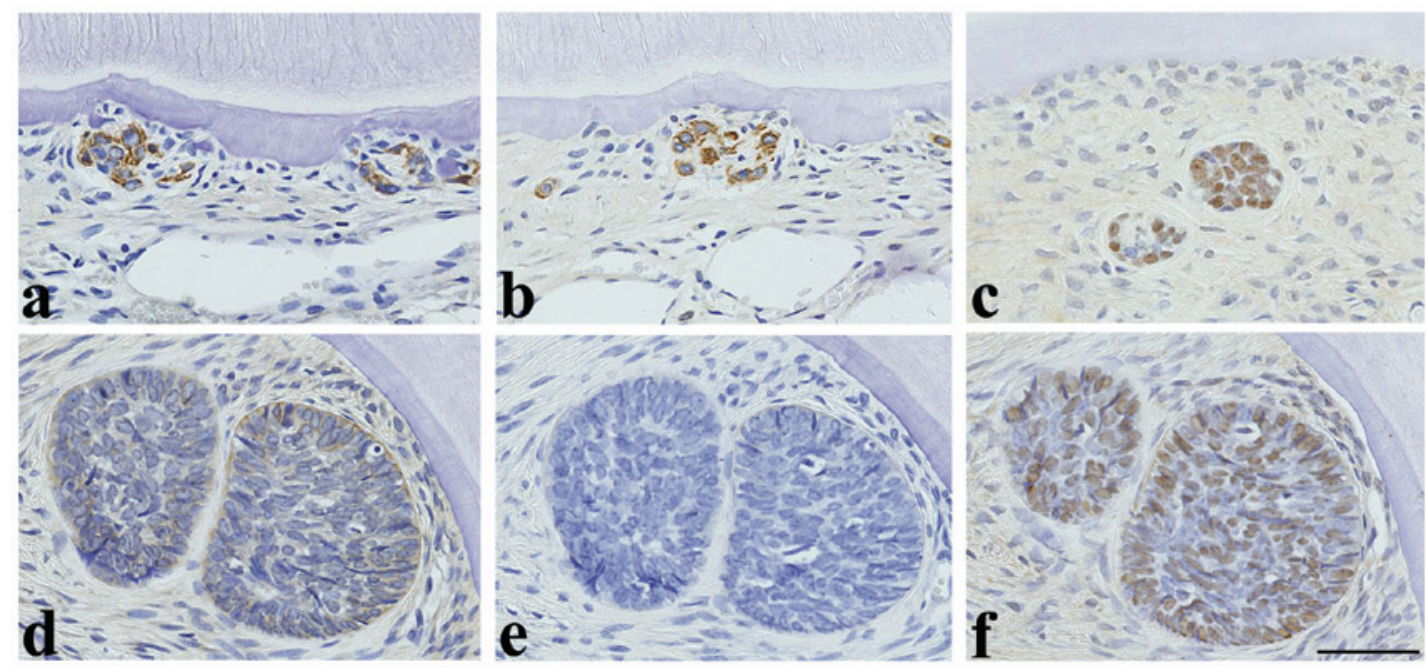

Figure 4. Immunoreactivities of epithelial rests of Malassez (ERM) in 30-week-old rats. (a and d) Tyrosine receptor kinase A (TrkA) immunohistochemistry in the control group and the $N$-methyl- $N$-nitrosourea (MNU)-treated group, respectively. (b and e) Cytokeratin 14 (CK14) immunohistochemistry in the control group and the MNU-treated group, respectively. (c and f) p63 immunohistochemistry in the control group and the MNU-treated group, respectively. Bar, $100 \mu \mathrm{m}$.

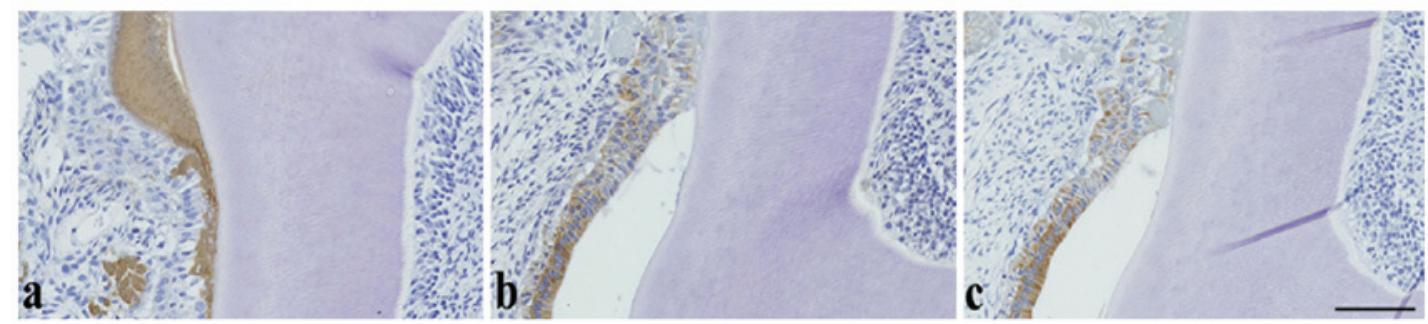

Figure 5. Immunoreactivities of odontogenic tumors induced by $N$-methyl- $N$-nitrosourea (MNU). (a) Amelogenin expression is observed in the cytoplasm of ameloblasts and secretory ameloblasts with tall columnar or cuboidal-shaped cells and in enamel-like tissue. (b) Tyrosine receptor kinase A (TrkA) expression is observed in the cytoplasm of ameloblasts and secretory ameloblasts with tall columnar or cuboidal-shaped cells. (c) Cytokeratin 14 (CK14) expression is observed in the cytoplasm of ameloblasts and secretory ameloblasts with tall columnar or cuboidal-shaped cells. Bar, $100 \mu \mathrm{m}$.

was significantly higher in the MNU-treated rats at each time point $(27.2,33.2$, and 24.7 at 12,18 , and 30 weeks, respectively) (Fig. 3a). Although there was no time-dependent increase in the number of ERM in the control or MNU-treated groups, MNU-treated rats had significantly larger ERM as compared with controls, and there was a time-dependent increase in the mean area of ERM (Fig. 3b) $\left(1108,2878\right.$, and $1268 \mu \mathrm{m}^{2}$ in control groups vs. 2428, 6061, and $9930 \mu \mathrm{m}^{2}$ in MNU-treated groups at 12,18 , and 30 weeks, respectively). This discrepancy between the number and area of ERM may be due to the fusion of enlarged ERM.

Immunohistochemical analysis of proliferative changes in molars. Regardless of MNU treatment, TrkA (Fig. 4a) and CK14 (Fig. 4b) immunoreactivity was observed in the cell cytoplasm and p63 immunoreactivity was observed in the cell nuclei (Fig. 4c) of the normal or relatively small ERM in the cervical and furcation regions of the molars, whereas amelogenin was negative (figure not shown). However, in the larger ERM observed following MNU treatment, TrkA and CK14 expression decreased or disappeared (Fig. 4d and 4e), whereas p63 expression (Fig. 4f) remained constantly high and amelogenin remained negative (figure not shown). In tumors, the expression of amelogenin (Fig. 5a), TrkA (Fig. 5b), and
CK14 (Fig. 5c) was detected in the cytoplasm of ameloblastlike cells and cells with tall columnar or cuboidal-shaped cells (secretory ameloblasts). Amelogenin was also expressed in enamel-like tissue, whereas p63 was negative. None of the antigens examined were expressed in odontoblast-like cells, dentin-like tissue or dental pulp-like mesenchymal cells.

\section{Discussion}

The present study examines changes in ERM as a precursor to odontogenic tumor development in the molar region following a single intraperitoneal injection of MNU into female Lewis rats. The number of ERM in MNU-treated rats was significantly higher at all time points as compared to control rats. The area of ERM in the MNU-treated rats was significantly larger than in the control rats, and it increased in a time-dependent manner. Finally, odontomas were observed in the molar and incisor regions in MNU-treated rats at 30 weeks of age.

Herrold was the first to induce odontogenic tumors in animals by exposure to MNU (18). Morphologically, MNU-induced tumors in Syrian hamsters resembled human ameloblastomas. Rats were later found to be susceptible to MNU-induced odontogenic tumors (15). MNU-induced odontogenic tumors in rodents are derived from the ameloblasts of 
the continuously erupting incisors, which is located adjacent to the root of the incisors $(12,14,17,26)$. A single injection of $150 \mathrm{mg} / \mathrm{kg}$ MNU causes death in approximately one third of rats in three weeks. Younger rats (6 weeks old or younger) were more susceptible to MNU than older rats (8 weeks old), and the susceptibility to the development of odontogenic lesions appears to end at 8 weeks of age (17). Local and multiple injections of ENU, a chemical closely related to MNU, coupled with mechanical injuries (incisor wounding) were found to accelerate the production of odontogenic tumors in the incisor region (9), which indicates that promoter stimuli may be required for a high yield of odontogenic tumors. Odontogenic tumors in the molar region occur in the cervical and furcation regions (but not in the apical region) where ERM always remain as a result of local administration of MNU mixed with alginate impression material to preserve MNU at the treatment site (26). Due to the similarity in locations of ERM and tumors, enlarged ERM may have acquired neoplastic characteristics. In contrast to tumors derived from ameloblasts of incisors, tumors derived from ERM of multi-rooted rat molars are similar to human tumors. However, to the best of our knowledge, it has not been previously reported that a single systemic administration of MNU causes odontogenic tumors in the molar region. In the present study, odontogenic tumors developed in the molar regions of female Lewis rats treated with MNU.

In general, odontogenic tumors are rarely encountered as spontaneous lesions in rodents $(1,5,43)$. In $\mathrm{Tg}$.AC mice, a mouse line created in the $\mathrm{FVB} / \mathrm{N}$ background by pronuclear injection of $\mathrm{v}$-Ha-ras oncogene, the incidences of odontogenic tumors (odontoma) were $16 \%$ in a 26 -week study and $35 \%$ in a 1 -year study $(5,44)$. In other transgenic mice, such as Hedgehog transcriptional effector Gli2-overexpressed mice, keratocystic-type odontogenic tumors arise from proliferative ERM (45). In Smad4-gene knockout mice (Smad4 ${ }^{\mathrm{Co} / \mathrm{Co}} \mathrm{OC}$-Cre), a similar type of odontogenic tumor arises from proliferative ERM (46). In the present study, the incidence of odontogenic tumors was $23 \%$ at the age of 30 weeks, which is compatible with the incidence in $\mathrm{Tg}$. AC mice.

Several genetic and molecular alterations appear to promote the development and progression of odontogenic tumors via multiple steps associated with tooth development, bone metabolism, and the malignant potential of tumors (6). In an in vitro study, ERM in combination with dental pulp cells generate enamel-like or dentin-like tissues in a similar manner to cervical loop epithelial cells (25). ERM have characteristics of stem/progenitor cells (36). Morphological continuity exists between ERM and the induced tumors in rats treated with local administration of MNU (26). TrkA (47), CK14 (25), and p63 (48) are markers for ERM in the periodontal ligament. p63 is a candidate epithelial stem cell marker in oral tissues (33-38). TrkA, CK14 and p63 were constantly expressed in small ERM of control rats, although TrkA and CK14 expression tended to decrease and disappear, respectively, in the larger ERM of MNU-treated rats. p63 was consistently detected in ERM of various sizes. However, ERM of various sizes were negative for amelogenin $(25,49)$. In the present study, the expression of TrkA and CK14 in large ERM was different from that in normal-sized ERM. The decreased levels of expression may corroborate with the change at the early transition or dedifferentiation stage of odontogenic carcinogenesis.
MNU-induced tumors were diagnosed as odontomas (complex type), since they contained dentin-like, enamel-like, and dental pulp-like mesenchymal cells $(2,30)$. Amelogenin is not detected in ERM (49), but it is detected in the cytoplasm of the tall columnar odontogenic epithelium, stellate reticulum-like cells, and their associated extracellular components together with enamel-like tissue in epithelial odontogenic tumors (50). In the present study, amelogenin was detected in the enamel-like matrix, in secretory ameloblasts adjacent to enamel-like matrix, and in cells assumed to be ameloblasts, but not in dentin-like or dental pulp-like mesenchymal cells. Amelogenin-CK14 interactions in ameloblasts occur during enamel formation (51) and cultured ERM express amelogenin and CK14 (25). Epithelial odontogenic tumors express various degrees of amelogenin and CK14 $(50,52)$. Co-expression of amelogenin and CK14 may be a characteristic of epithelial odontogenic tumors, indicating that these tumors have ameloblastic differentiation or odontogenic epithelial properties. In addition, TrkA was expressed in tumor cells with ameloblastic differentiation, although the reasons for this are unclear. p63 is essential for tooth development (53) and is expressed in dental germ cells (35), ameloblasts (37), and various odontogenic tumors including ameloblastomas $(48,54)$. Although p63 was consistently detected in ERM of various sizes, the compound odontomas in the present study were p63-negative.p63 expression correlates to malignancy in odontogenic tumors (48). In conclusion, a single intraperitoneal injection of MNU resulted in the development of odontogenic tumors with mixed enamel and dentin differentiation (complex odontomas) in the molar region, and these tumors may have been derived from ERM. Detailed mechanistic investigations of the molecular basis of odontogenic carcinogenesis are required to further elucidate the relationship between ERM and odontogenic tumors in rodents and humans.

\section{Acknowledgements}

The authors thank Ms. T. Akamatsu for her technical assistance and Ms. A. Shudo for manuscript preparation. This work was supported in part by a grant-in-aid for Scientific Research (C) (22591954)

\section{References}

1. Brown HR and Hardisty JF: Oral cavity, esophagus, and stomach. In: Pathology of the Fischer Rat: Reference and Atlas. Boorman GA, Montgomery CA Jr and MacKenzie WF (eds). Academic Press, San Diego, pp9-30, 1990.

2. Long PH and Leininger JR: Teeth. In: Pathology of the Mouse: Reference and Atlas. Maronpot RR, Boorman GA and Gaul BW (eds). Cache River Press, Vienna, pp13-28, 1999.

3. Abe T and Miyajima H: Effect of drugs on developing enamel in rat incisors. J Toxicol Pathol 3: 245-256, 1990. (Abstract in English, text in Japanese)

4. Kuijpers MH, van de Kooij AJ and Slootweg PJ: The rat incisor in toxicologic pathology. Toxicol Pathol 24: 346-360, 1996.

5. Weber K: Induced and spontaneous lesions in teeth of laboratory animals. J Toxicol Pathol 20: 203-213, 2007.

6. Kumamoto H: Molecular pathology of odontogenic tumors J Oral Pathol Med 35: 65-74, 2006.

7. Takeda Y: Histogenesis and clinical pathology of odontogenic tumors. Dent J Iwate Med Univ 31: 143-152, 2006 (In Japanese).

8. Edwards MB: Some effects of N-methylnitrosourea on the oral and odontogenic tissues of the Syrian golden hamster. Arch Oral Biol 23: 515-524, 1978. 
9. Maeda H, Kameyama Y, Fujita K, Sato E and Mizutani M Experimental odontogenic tumors produced by ethylnitrosourea injections and mechanical injuries. J Oral Pathol Med 20: 296-299, 1991

10. Satoh H, Usegi Y, Kawabata T, Mori K, Fujii F and Kashimoto Y: Morphological classification of dental lesions induced by various antitumor drugs in mice. Toxicol Pathol 29: 292-299, 2001.

11. Azuma T and Komori A: An experimental study of odontogenic tumors in rats. Induction of tumors by gastric intubation of MNU. Jpn J Oral Biol 27: 631-639, 1985.

12. Berman JJ and Rice JM: Odontogenic tumours produced in Fischer rats by a single intraportal injection of methylnitrosourea. Arch Oral Biol 25: 213-220, 1980.

13. Eblin H, Barbachan JJD, Do Valle JGC and De Oliveira LY: N-Methyl-N-nitrosourea-induced odontogenic neoplasms in rats. J Dent Res 52: 177, 1973

14. Eisenberg E, Murthy AS, Vawter GF and Krutchkoff DJ: Odontogenic neoplasms in Wistar rats treated with N-methylnitrosourea. Oral Surg Oral Med Oral Pathol 55: 481-486, 1983.

15. Gardner DG: Experimentally induced ameloblastomas: a critical review. J Oral Pathol Med 21: 337-339, 1992.

16. Leaver DD, Swann PF and Magee PN: The induction of tumors in the rat by a single oral dose of $\mathrm{N}$-nitrosomethylurea. $\mathrm{Br} \mathrm{J}$ Cancer 223: 177-187, 1969

17. Smulow JB,Konstantinidis A and Sonnenschein C: Age-dependent odontogenic lesions in rats after a single i.p. injection of N-nitrosoN-methylurea. Carcinogenesis 4: 1085-1088, 1983.

18. Herrold KM: Odontogenic tumors and epidermoid carcinomas of the oral cavity. An experimental study in Syrian hamsters. Oral Surg Oral Med Oral Pathol 25: 262-272, 1968.

19. Kohgo T: An experimental study of odontogenic tumors in hamsters by N-nitrosomethylurea. J Stomatol Soc Jpn 39: 191-212, 1972

20. Kohgo T, Iizuka T and Shindoh M: Pathological evaluation of the effects of intentional disocclusion and overloading occlusion in odontogenesis disorders in N-methylnitrosourea-treated hamsters. Toxicol Pathol 27: 226-232, 1999.

21. Wentz FM, Weinmann JP and Schour I: The prevalence, distribution, and morphologic changes of the epithelial remnants in the molar region of the rat. J Dent Res 29: 637-646, 1950.

22. Bykov VL: Epithelial cell rests of Malassez: tissue, cell, and molecular biology. Morfologiia 124: 95-103, 2003 (In Russian).

23. Kat PS, Sampson WJ, Wilson DF and Wiebkin OW: Distribution of the epithelial rests of Malassez and their relationship to blood vessels of the periodontal ligament during rat tooth development. Aust Orthod J 19: 77-86, 2003.

24. Huang X, Bringas P, Slavkin HC and Chai Y: Fate of HERS during tooth root development. Dev Biol 334: 22-30, 2009.

25. Shinmura Y, Tsuchiya S, Hata K and Honda MJ: Quiescent epithelial cell rests of Malassez can differentiate into ameloblastlike cells. J Cell Physiol 217 : 728-738, 2008.

26. Hamamoto $Y$, Hamamoto $N$, Nakajima $T$ and Ozawa $H$ : Morphological changes of epithelial rests of Malassez in rat molars induced by local administration of $\mathrm{N}$-methylnitrosourea. Arch Oral Biol 43: 899-906, 1998

27. Rincon JC, Young WG and Bartold PM: The epithelial cell rests of Malassez - a role in periodontal regeneration? J Periodontal Res 41: 245-252, 2006.

28. Yagoto M, Shimojyo Y, Uotani Y, et al: Methodology of histological preparation of rat teeth tissue including incisor and molar. Jpn J Histotech 9: 29-32, 2000 (In Japanese).

29. Long PH, Leininger JR and Lieuallen WG: Non-proliferative lesions of bone, cartilage, tooth, and synovium in rats. In: Guides for Toxicologic Pathology. STP/ARP/AFIP, Washington, D.C., 1996.

30. Long PH, Leininger JR, Nold JB and Lieuallen WG: Proliferative lesions of bone, cartilage, tooth, and synovium in rats. In: Guides for Toxicologic Pathology. STP/ARP/AFIP, Washington, D.C., 1996.

31. Kurihara H, Shinohara H, Yoshino H, Takeda K and Shiba $H$ : Neurotrophins in cultured cells from periodontal tissues. J Periodontol 74: 76-84, 2003.

32. Woodnutt DA and Byers MR: Morphological variation in the tyrosine receptor kinase A- immunoreactive periodontal ligament. Arch Oral Biol 46: 163-171, 2001.
33. Brikic A, Mutlu S, Kocak-Berberoglu H and Olgac V: Pathological changes and immunoexpression of p63 gene in dental follicles of asymptomatic impacted lower third molars: an immunohistochemical study. J Craniofac Surg 21: 854-857, 2010.

34. Casasco M, Cornaglia AI, Riva F, Calligaro A and Casasco A Expression of $\mathrm{p} 63$ transcription factor in ectoderm-derived oral tissues. Int J Anat Embryol 111: 125-131, 2006

35. Kock M, Nolting D, Kjaer KW, Hansen BF and Kjaer I: Immunohistochemical expression of p63 in human prenatal tooth primordia. Acta Odontol Scand 63: 253-257, 2005

36. Nam H, Kim J, Park J, et al: Expression profile of the stem cell markers in human Hertwig's epithelial root sheath/epithelial rests of Malassez cells. Mol Cells 31: 355-360, 2011

37. Rufini A, Weil M, McKeon F, Barlattani A, Melino G and Candi E: p63 protein is essential for the embryonic development of vibrissae and teeth. Biochem Biophys Res Commun 340: 737-741, 2006.

38. Xiong J, Gronthos S, Krzysztof M and Bartold PM: Characterisation and stem-cell properties of epithelial cell rests of Malassez. Abstract in IADR/AADR/CADR 89th General Session and Exhibition, San Diego, USA, 2011.

39. Uchida $T$, Tanabe $T$ and Fukae M: Immunocytochemical localization of amelogenins in the deciduous tooth germs of the human fetus. Arch Histol Cytol 52: 543-552, 1989.

40. Uchida T, Tanabe T, Fukae M et al: Immunochemical and immunohistochemical studies, using antisera against porcine $25 \mathrm{kDa}$ amelogenin, $89 \mathrm{kDa}$ enamelin and the 13-17 kDa nonamelogenins, on immature enamel of the pig and rat. Histochemistry 96: $129-138,1991$

41. Hasegawa N, Kawaguchi H, Ogawa T, Uchida T and Kurihara H: Immunohistochemical characteristics of epithelial cell rests of Malassez during cementum repair. J Periodont Res 38: 51-56, 2003.

42. Nishio C, Wazen R, Kuroda S, Moffatt P and Nanci A: Disruption of periodontal integrity induces expression of apin by epithelial cell rests of Malassez. J Periodont Res 45: 709-713, 2010.

43. Lewis DJ, Cherry CP and Gibson WA: Ameloblastoma (adamantinoma) of the mandible in the rat. J Comp Pathol 90: 379-384, 1980.

44. Wright JT, Hansen L, Mahler J, Szczesniak C and Spalding JW: Odontogenic tumors in the v-Ha-ras (TGAC) transgenic mouse. Archs Oral Biol 40: 631-638, 1995.

45. Grachtchouk M, Liu J, Wang A, et al: Odontogenic keratocysts arise from quiescent epithelial rests and are associated with deregulated hedgehog signaling in mice and humans. Am J Pathol 169: 806-814, 2006.

46. Gao Y, Yang G, Weng T, et al: Disruption of Smad4 in odontoblasts causes multiple keratocytic odontogenic tumors and tooth malformation in mice. Mol Cell Biol 29: 5941-5951, 2009.

47. Yamashiro T, Fujiyama K, Fukunaga T, Wang $Y$ and Takano-Yamamoto T: Epithelial rests of Malassez express immunoreactivity of TrkA and its distribution is regulated by sensory nerve innervation. J Histochem Cytochem 48: 979-984, 2000.

48. Lo Muzio L, Santarelli A, Caltabiano R, et al: p63 expression correlates with pathological features and biological behaviour of odontogenic tumours. Histopathology 49: 211-214, 2006.

49. Hamamoto Y, Nakajima T, Ozawa H and Uchida T: Production of amelogenin by enamel epithelium of Hertwig's root sheath. Oral Surg Oral Med Oral Pathol Oral Radiol Endod 81: 703-709, 1996.

50. Kumamoto H, Yoshida M and Ooya K: Immunohistochemical detection of amelogenin and cytokeratin 19 in epithelial odontogenic tumors. Oral Dis 7: 171-176, 2001.

51. Ravindranath RM, Tam WY, Bringas P Jr, Santos V and Fincham AG: Amelogenin-cytokeratin 14 interaction in ameloblasts during enamel formation. J Biol Chem 276: 36586-36597, 2001.

52. Crivelini MM, de Araújo VC, de Sousa SO and de Araújo NS: Cytokeratins in epithelia of odontogenic neoplasms. Oral Dis 9: $1-6,2003$.

53. Rufini A, Barlattani A, Docimo R, et al: p63 in tooth development. Biochem Pharmacol 82: 1256-1261, 2011.

54. Kumamoto H, Ohki K and Ooya K: Expression of p63 and p73 in ameloblastomas. J Oral Pathol Med 34: 220-226, 2005. 